\title{
Performance of Commercial Blood Tests for the Diagnosis of Latent Tuberculosis Infection in Children and Adolescents
}

\author{
Barbara Maria Bergamini, MDa, Monica Losi, PhD ${ }^{b}$, Francesca Vaienti, MDa, Roberto D'Amico, PhDc, Barbara Meccugni, BScd, \\ Marisa Meacci, BScd, Donatella De Giovanni, MDa, Fabio Rumpianesi, MDd, Leonardo M. Fabbri, MD ${ }^{b}$, Fiorella Balli, MDa, \\ Luca Richeldi, MD, PhDb
}

Departments of aPediatrics, bRespiratory Diseases, and CStatistics, University of Modena and Reggio Emilia, Modena, Italy; dMicrobiology and Virology Laboratory, Policlinico Hospital of Modena, Modena, Italy

The authors have indicated they have no financial relationships relevant to this article to disclose.

\section{What's Known on This Subject}

Diagnosis of LTBl in children is clinically relevant; however, the standard TST might have false-negative results in this age group. IGRAs hold promise to be more accurate than the skin test.

\section{What This Study Adds}

Different IGRAs perform differently, particularly among children $<4$ years of age, in terms of rates of indeterminate results: the ELISA-based assays are negatively affected by younger age compared with the ELISPOT-based test.

\section{ABSTRACT}

BACKGROUND. The accurate diagnosis of latent tuberculosis infection reduces the risk of progression to severe disseminated disease. However, in young children, a major limitation of the standard tuberculin skin test is that false-negative results cannot be detected. The new interferon- $\gamma$ release assays QuantiFERON-TB Gold (Cellestis Carnegie Victoria, Australia), QuantiFERON-TB In-Tube (Cellestis), and T-SPOT.TB (Oxford Immunotec, Abingdon, United Kingdom) show promise of greater accuracy, but they may also be affected by impaired cellular immunity, resulting in indeterminate results (ie, insufficient response in positive-control wells).

OBJECTIVE. To evaluate the impact of age on the performance of interferon- $\gamma$ release assays when used in a routine hospital setting among children tested for suspected active or latent TB infection.

METHODS. We retrospectively studied 496 children 0 to 19 years of age who had been tested with the tuberculin skin test and at least 1 interferon- $\gamma$ release assay: 181 with QuantiFERON-TB Gold and 315 with QuantiFERON-TB In-Tube. In 154 of the children, paired interferon- $\gamma$ release assay testing was available: 87 with QuantiFERON-TB Gold/T-SPOT.TB and 67 with QuantiFERON-TB In-Tube/T-SPOT.TB.

RESULTS. Compared with T-SPOT.TB, the rates of indeterminate results were significantly higher for both QuantiFERON-TB Gold and QuantiFERON-TB In-Tube. QuantiFERON-TB Gold and QuantiFERON-TB In-Tube also gave indeterminate results more frequently in children $<4$ years of age than in those $\geq 4$ years of age. Indeterminate results were associated with younger age for both QuantiFERON-TB Gold and QuantiFERON-TB In-Tube but not for T-SPOT.TB. Considering age as a binary variable $(<4$ and $\geq 4$ years of age), a significantly higher concentration of phytohaemagglutinin-produced interferon- $\gamma$ was observed in older children with both QuantiFERON-TB Gold and QuantiFERON-TB In-Tube.

CONCLUSIONS. Different blood tests for the diagnosis of latent tuberculosis infection in children seem to perform differently, because both QuantiFERON-TB tests were more likely than T-SPOT.TB to give indeterminate results in children <4 years of age. Pediatrics 2009;123:e419-e424 www.pediatrics.org/cgi/doi/10.1542/ peds.2008-1722

doi:10.1542/peds.2008-1722

Key Words

tuberculosis infection, diagnosis, children,

interferon- $\gamma$

Abbreviations

IGRA-interferon- $\gamma$ release assay

IFN- $\gamma$-interferon- $\gamma$

ELISA—enzyme-linked immunosorbent assay

ELISPOT—enzyme-linked immunospot

QFT-G_QuantiFERON-TB Gold

QFT-IT-QuantiFERON-TB Gold In-Tube

TST-tuberculin skin test

LTBI-latent tuberculosis infection

TB-tuberculosis

ESAT-6 - early secretory antigen target 6

CFP10 - culture filtrate protein 10

SFU—spot-forming unit

OR-odds ratio

$\mathrm{Cl}$-confidence interval

Accepted for publication Dec 4, 2008

Address correspondence to Luca Richeldi, MD, PhD, University of Modena and Reggio Emilia, Department of Respiratory Diseases, Policlinico Hospital of Modena, Via del Pozzo 71,41100 Modena, Italy. E-mail luca.richeldi@ unimore.it.

PEDIATRICS (ISSN Numbers: Print, 0031-4005; Online, 1098-4275). Copyright $\odot 2009$ by the American Academy of Pediatrics

TN YOUng CHILDREN, the risk of progression from latent tuberculosis infection (LTBI) to active tuberculosis (TB) Lis inversely related to age; without specific treatment, approximately one half of infants and one fifth of older children develop active TB up to 2 years after infection. ${ }^{1}$ In particular, children $<4$ years of age fail to contain the spread of intracellular pathogens as a consequence of an impaired T-cell response. ${ }^{2-4}$ Therefore, this age group is considered a priority in the strategies to control TB worldwide. The century-old tuberculin skin test (TST) is still the standard method for diagnosing LTBI, but it has drawbacks, such as low specificity in 


\begin{tabular}{|c|c|c|c|c|c|c|}
\hline & $\begin{array}{l}\text { QFT-G only } \\
(n=181)\end{array}$ & $\begin{array}{c}\text { QFT-G and } \\
\text { T-SPOT.TB } \\
(n=87)\end{array}$ & $\begin{array}{l}\text { QFT-IT only } \\
(n=315)\end{array}$ & $\begin{array}{c}\text { QFT-IT and } \\
\text { T-SPOT.TB } \\
(n=67)\end{array}$ & $\begin{array}{c}\text { T-SPOT.TBa } \\
(n=154)\end{array}$ & $N=496$ \\
\hline Mean age, $n(S D)$ & $10.5(5.9)$ & $10.2(5.9)$ & $11.5(5.5)$ & $7.9(6.4)$ & $9.2(6.0)$ & $11.1(5.7)$ \\
\hline$<4 \mathrm{y}, n(\%)$ & $39(21.6)$ & $21(24.1)$ & $65(20.6)$ & $36(53.7)$ & $57(37.0)$ & $104(21.0)$ \\
\hline$\geq 4 \mathrm{y}, n(\%)$ & $142(78.4)$ & $66(75.9)$ & $250(79.4)$ & $32(47.8)$ & $98(63.6)$ & $392(79.0)$ \\
\hline Boys, $n(\%)$ & $88(48.6)$ & $40(46.0)$ & $124(39.4)$ & $22(32.8)$ & $62(40.3)$ & $212(42.7)$ \\
\hline BCG-vaccinated, $n$ (\%) & 54 (41.9) & $24(36.9)$ & $139(49.1)$ & $5(8.1)$ & $29(18.8)$ & $193(38.9)$ \\
\hline \multicolumn{7}{|l|}{ Reason for testing, $n(\%)$} \\
\hline Recent immigration from area with endemic TB & $78(43.1)$ & $28(32.2)$ & $126(40)$ & $3(4.5)$ & $31(20.1)$ & $204(41.1)$ \\
\hline Recent contact with a pulmonary TB case & $91(50.3)$ & $49(56.3)$ & $176(55.9)$ & $60(89.6)$ & $109(70.8)$ & $267(53.8)$ \\
\hline Suspected active TB & $12(6.6)$ & $10(11.5)$ & $13(4.1)$ & $4(6.0)$ & $14(9.1)$ & $25(5.1)$ \\
\hline \multicolumn{7}{|l|}{ Place of birth, $n(\%)$} \\
\hline Africa & $47(26.0)$ & $20(23.0)$ & $81(25.6)$ & $7(10.4)$ & $27(17.5)$ & $128(25.8)$ \\
\hline Middle East & $29(16.0)$ & $23(26.4)$ & 34 (10.9) & $3(4.5)$ & $26(16.9)$ & $63(12.8)$ \\
\hline Eastern Europe & $33(18.2)$ & $13(14.9)$ & $56(17.6)$ & $3(4.5)$ & $16(10.4)$ & $89(17.8)$ \\
\hline Asia & $14(7.7)$ & $3(3.4)$ & $17(5.4)$ & $3(4.5)$ & $6(3.9)$ & $31(6.3)$ \\
\hline South America & $6(3.3)$ & $0(0)$ & $5(1.6)$ & $0(0)$ & $0(0)$ & $11(2.2)$ \\
\hline Western Europe & $52(28.7)$ & $28(32.2)$ & $122(38.8)$ & $51(76.1)$ & $79(51.3)$ & $174(35.1)$ \\
\hline
\end{tabular}

a From dual tests with a QFT.

Mycobacterium bovis BCG-vaccinated persons and low sensitivity among patients with an impaired cellular immune system. ${ }^{5}$ Paradoxically, the TST has more false-negative results in populations with higher risk of TB reactivation; $\sim 10 \%$ of children with cultureconfirmed TB do not react to the TST at the time of diagnosis. ${ }^{1,6}$

A new generation of LTBI diagnostics, the interferon- $\gamma($ IFN- $\gamma$ ) release assays (IGRAs), seem to be significantly more accurate than the skin test for the diagnosis of LTBI., $3,7,8$ Three commercially available IGRAs have been approved for clinical use by the Food and Drug Administration. T-SPOT.TB (Oxford Immunotec Ltd, Abingdon, United Kingdom) is based on the enzyme-linked immunospot (ELISPOT) technology, whereas QuantiFERON-TB Gold (QFT-G) and QuantiFERON-TB Gold In-Tube (QFT-IT) (Cellestis Ltd, Carnegie Victoria, Australia) are based on the enzyme-linked immunosorbent assay (ELISA). These tests share common features but also have some technical differences. ${ }^{7}$ IGRAs have already been shown to be significantly more specific than the TST, ${ }^{8}$ and preliminary evidence from 2 recent longitudinal studies indicates that IGRAs may have higher positive predictive values for development of future TB, compared with the skin test.9,10 The performance of IGRAs for diagnosis in children has not been extensively explored. ${ }^{3,8,11}$ In particular, the rate of indeterminate results, that is, an insufficient response in positivecontrol wells, is a clinically relevant issue. Few studies have been published on this topic ${ }^{12-18}$ and fewer have explored the relationship between IFN- $\gamma$ production and age. . $3,16,19^{19}$ Therefore, we analyzed the performance of IGRAs under routine conditions in a large pediatric population to investigate the frequency of indeterminate results and the potential effect of age on these diagnostic tests.

\section{PATIENTS AND METHODS}

\section{Study Population}

We retrospectively evaluated all patients tested with at least 1 IGRA at the pediatric department of the University Hospital of Modena (Italy) between January 2004 and July 2007. Testing was performed because of suspected TB infection as a result of recent immigration $(\leq 2$ years), household contact with a person with active pulmonary $\mathrm{TB}$, or suspected pulmonary or lymph-nodal TB. Parents of the children consented in advance to have sensitive clinical data collected for future research. Children were tested for clinical purposes with 1 or more IGRAs, according to the availability of the assays: QFT-G (alone or combined with T-SPOT.TB) until December 2004 and QFT-IT (alone or combined with T-SPOT.TB) afterward (Table 1).

\section{Procedures}

Demographic and clinical data, including birthplace, BCG vaccination status (vaccination certificate and/or the presence of the typical scar), TST result, and reasons for testing were collected by using standard clinical forms. All enrolled subjects underwent a TST with 5 tuberculin units of purified protein derivative (Biocine test PPD, Chiron, Siena, Italy), according to the intradermal Mantoux method. TST results were interpreted by trained physicians according to current pediatric guidelines (ie, the test was considered positive if the skin induration was $\geq 5 \mathrm{~mm}$ in diameter in children with suspected active TB or recent TB contact or $\geq 10 \mathrm{~mm}$ in children who were $<4$ years of age or were tested because of recent immigration from an area in which TB is endemic, regardless of BCG status). ${ }^{11,20}$ Peripheral blood samples for IGRAs were drawn and transferred within 3 hours to the laboratory, where they were processed by trained technicians and interpreted according to the recommendations of manufacturers. 
The T-SPOT.TB test was performed on peripheral blood mononuclear cells separated by centrifugation from a sample of peripheral venous blood $(4 \mathrm{~mL}$ for children $\leq 6$ years of age and $8 \mathrm{~mL}$ for older children). Peripheral blood mononuclear cells were plated $(2.5 \times$ $10^{5}$ cells per well) on a plate precoated with an antiIFN- $\gamma$ antibody. The test included (1) 2 separate antigen wells containing either early secretory antigen target 6 (ESAT-6) or culture filtrate protein 10 (CFP10) (Mycobacterium tuberculosis-specific antigens); (2) a positivecontrol well containing phytohaemagglutinin; and (3) a negative-control well containing neither mitogen nor antigen. Plates were incubated overnight at $37^{\circ} \mathrm{C}$ in an atmosphere of $5 \% \mathrm{CO}_{2}$; after washing, plates were developed with a conjugate against the IFN- $\gamma$ antibody, and spot-forming units (SFUs) were counted by using an automated ELISPOT reader (AID, Strassberg, Germany). SFU values in the negative-control well were subtracted from SFU values in the mitogen and antigen wells.

If the number of SFUs in the negative-control well was $<5$, T-SPOT.TB results were defined as ( 1 ) positive if either antigen well had at least 6 SFUs more than the negative control, regardless of the response in the positive control; (2) negative if the number of SFUs was $<6$ in both antigen wells and if the positive-control well had $\geq 20$ SFUs; and (3) indeterminate if the positivecontrol well had $<20$ SFUs and both antigen wells had $<6$ SFUs. Furthermore, if the number of SFUs in the negative-control well was $\geq 6$, T-SPOT.TB results were defined as positive if either antigen well had at least twice the number of SFUs as the negative control, regardless of the response in the positive control. If the number of SFUs in the negative-control well was $\geq 10$, T-SPOT.TB results were defined as a technical error and not considered in the statistical analysis.

The QFT-G test consisted of a negative-control well ( 1 $\mathrm{mL}$ of whole blood without antigens or mitogen), a positive-control well ( $1 \mathrm{~mL}$ of whole blood stimulated with phytohaemagglutinin), and 2 antigen wells (1 mL of whole blood in each well) stimulated with ESAT-6 or CFP10. Whole blood specimens were incubated overnight at $37^{\circ} \mathrm{C}$ in a humidified atmosphere. Plasma aliquots were harvested, and IFN- $\gamma$ concentrations were determined by using an ELISA with an automated system ELISA reader (Dinex Technologies, West Sussex, United Kingdom) and expressed as IUs. QFT-G results were defined as ( 1 ) positive if the concentration of IFN- $\gamma$ in the antigen well after stimulation with ESAT- 6 or CFP10 was $\geq 0.35 \mathrm{IU} / \mathrm{mL}$ after subtraction of the value of IFN- $\gamma$ in the negative control, regardless of the response in the positive control; (2) negative if the response to the specific antigens after subtraction of the value of the negative-control well was $<0.35 \mathrm{IU} / \mathrm{mL}$ and the IFN- $\gamma$ concentration in the positive-control well was $\geq 0.50$ $\mathrm{IU} / \mathrm{mL}$; and (3) indeterminate if the concentration of IFN- $\gamma$ in both antigen wells was negative $(<0.35 \mathrm{IU} / \mathrm{mL})$ and the value of the positive control was $<0.50 \mathrm{IU} / \mathrm{mL}$. If the concentration of IFN- $\gamma$ in the negative-control well was $\geq 2 \mathrm{UI} / \mathrm{mL}$, the test was considered a technical error and excluded from statistical analysis.

For the QFT-IT test, $1 \mathrm{~mL}$ of peripheral venous blood
TABLE 2 Characteristics of the Study Population $(N=496)$

\begin{tabular}{lc}
\hline \multicolumn{1}{c}{ Characteristic } & Value \\
\hline Mean age (SD) & $11.1(5.7)$ \\
$<4 \mathrm{y}, n(\%)$ & $104(21.0)$ \\
$\geq 4 \mathrm{y}, n(\%)$ & $392(79.0)$ \\
Boys, $n(\%)$ & $212(42.7)$ \\
BCG-vaccinated, $n$ (\%) & $193(38.9)$ \\
Reason for testing, $n$ (\%) & \\
$\quad$ Recent immigration from area with endemic TB & $204(41.1)$ \\
Recent contact with a pulmonary TB patient ${ }^{2}$ & $267(53.8)$ \\
Suspected active TB & $25(5.1)$ \\
Place of birth, $n$ (\%) & \\
Africa & $128(25.8)$ \\
Middle East & $63(12.8)$ \\
Eastern Europe & $89(17.8)$ \\
Asia & $31(6.3)$ \\
South America & $11(2.2)$ \\
Western Europe & $174(35.1)$ \\
\hline
\end{tabular}

a Fifty-three (19.8\%) subjects were also recent immigrants.

was collected into each of 3 vacutainer tubes precoated with either saline (negative control), phytohaemagglutinin (positive control), or ESAT-6, CFP10, and TB7.7. Results were interpreted as for the QFT-G test, except cutoff for the negative-control well was set at $8.0 \mathrm{IU} / \mathrm{mL}$.

\section{Statistical Analysis}

Binary data were expressed as percentages, continuous normally distributed data were expressed as means \pm $\mathrm{SD}$, and as medians otherwise. Comparisons between percentages for paired data were conducted by a McNemar test, whereas the $t$ test was used for means (for variables not normally distributed we did compare medians). The association between indeterminate results and age was evaluated by logistic regression. The estimate of odds ratio (OR) and its relative $95 \%$ confidence interval (95\% CI) were calculated. The association between age and the concentration of phytohaemagglutinin-induced IFN- $\gamma$ in both QFT assays or the number of SFUs in T-SPOT.TB, which were previously log transformed because of their distributional deviation from normality, was assessed by linear regression. Data were analyzed for children $<4$ and $\geq 4$ years of age based on increased susceptibility to TB in younger children. Diagnostic agreement between tests was assessed by using Cohen's $\kappa$ statistics. Level of significance was set to .05 . Analyses were performed by using Stata 10.0 software (Stata Corp, College Station, TX).

\section{RESULTS}

Of 501 children tested, 5 were excluded because of possible technical errors (ie, high background in the negative-control well): 2 with QFT-G, 2 with QFT-IT, and 1 with T-SPOT.TB. None of the remaining 496 children had a known or clinically relevant impairment of cell immunity or any indication for HIV testing. Characteristics of the study population are reported in Table 2 .

\section{Testing for LTBI}

A positive IGRA result was obtained in 34 (18.8\%) of the 181 children tested with QFT-G, 54 (17.1\%) of the 315 
TABLE 3 Rates of Positive Results With IGRAs and the TST, Correlated With Reasons for Testing

\begin{tabular}{|c|c|c|c|c|c|c|c|c|c|c|c|c|}
\hline & \multicolumn{4}{|c|}{ QFT-G } & \multicolumn{4}{|c|}{ QFT-IT } & \multicolumn{4}{|c|}{ T-SPOT.TBa } \\
\hline & $n$ & $\begin{array}{c}\text { Positive, } \\
n(\%)\end{array}$ & $\begin{array}{c}\text { TST-positive, } \\
n(\%)\end{array}$ & $\kappa$ & $n$ & $\begin{array}{c}\text { Positive, } \\
n(\%)\end{array}$ & $\begin{array}{c}\text { TST-positive, } \\
n(\%)\end{array}$ & $\kappa$ & $n$ & $\begin{array}{c}\text { Positive, } \\
n(\%)\end{array}$ & $\begin{array}{c}\text { TST-positive, } \\
n(\%)\end{array}$ & $\kappa$ \\
\hline All & 181 & 34 (18.8) & $58(32.0)$ & 0.43 & 315 & $54(17.1)$ & 74 (23.5) & 0.35 & 154 & $22(14.3)$ & $28(18.2)$ & 0.52 \\
\hline Recent immigrants & 78 & $21(26.9)$ & 35 (44.9) & 0.26 & 126 & 39 (30.9) & $46(36.5)$ & 0.16 & 31 & $11(35.5)$ & $13(41.9)$ & 0.18 \\
\hline Recent TB contact & 91 & $10(11.0)$ & $18(19.8)$ & 0.57 & 176 & $11(6.3)$ & $21(11.9)$ & 0.45 & 109 & $5(4.6)$ & $9(8.3)$ & 0.69 \\
\hline Suspected active TB & 12 & $3(25.0)$ & $5(41.7)$ & 0.60 & 13 & $4(30.8)$ & $7(53.8)$ & 0.66 & 14 & $6(42.9)$ & $6(42.9)$ & 0.38 \\
\hline
\end{tabular}

a From dual tests with a QFT.

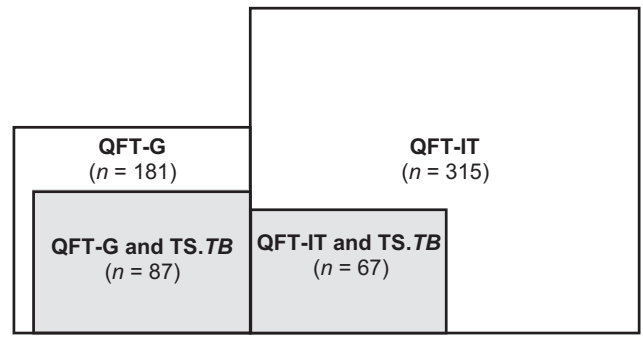

FIGURE 1

IGRA testing in the study population. TS.TB indicates T-SPOT.TB.

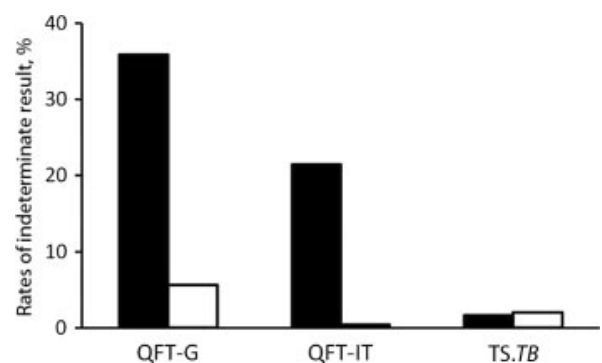

FIGURE 2

Rates of indeterminate IGRA results in children $<4$ years of age (black bars) or $\geq 4$ years of age (white bars) who were tested with QFT-G $(n=181)$, QFT-IT $(n=315)$, or T-SPOT.TB $(n=154)$.

tested with QFT-IT, and $22(14.3 \%)$ of the 154 tested with both a QFT and T-SPOT.TB test (Table 3). Both IGRAs showed a lower agreement with the TST in BCGvaccinated subjects than in the non-BCG vaccinated subjects, although the difference was statistically significant for QFT-G and QFT-IT only (data not shown). More positive IGRA results were observed among recent immigrants and those suspected of having active TB, whereas agreement with the TST was much lower among recent immigrants (Table 3 ).

\section{Testing for Response to Mitogen}

One hundred eighty-one children were tested with QFT-G and 315 with QFT-IT. For 154 of the 496 patients, paired results with T-SPOT.TB were also available: 87 with QFT-G/T-SPOT.TB and 67 with QFT-IT/T-SPOT.TB (Table 1 and Fig 1). In children with paired tests, indeterminate results were significantly more frequent with QFT-G or QFT-IT than with T-SPOT.TB (12.6\% vs $2.3 \%$ $[P=.0225]$ and $16.4 \%$ vs $1.5 \%[P=.0063]$, respectively).

The rates of indeterminate IGRA results were higher in children $<4$ years of age than in children $\geq 4$ years of
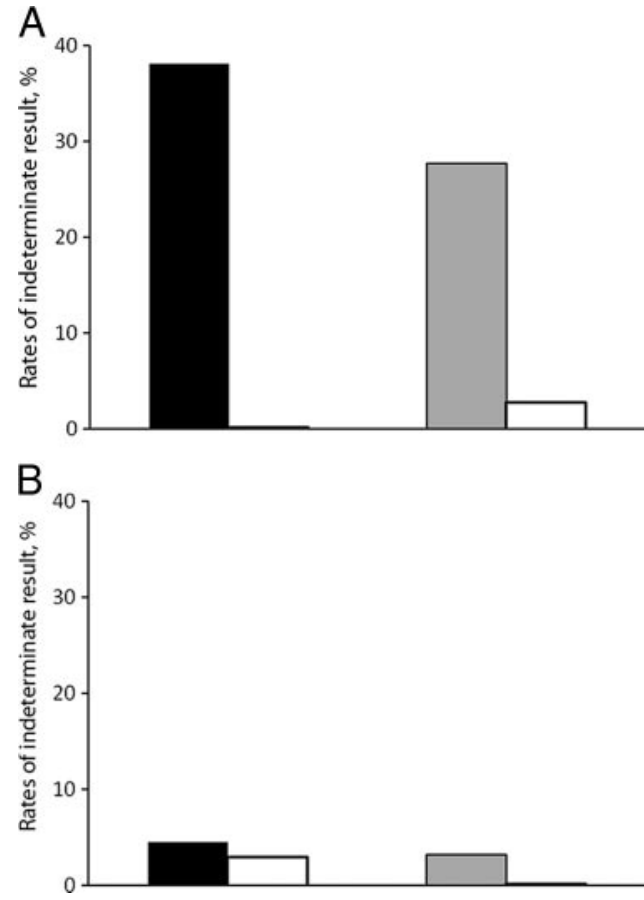

FIGURE 3

Rates of indeterminate IGRA results in paired testing of children $<4$ years of age (A) or $\geq 4$ years of age (B). Black columns, QFT-G; gray columns, QFT-IT; white columns, T-SPOT.TB.

age for both QFT-G $(35.9 \%$ vs $5.6 \%[P<.001])$ and QFT-IT $(21.5 \%$ vs $0.4 \%[P<.001])$, whereas no statistically significant difference was observed for T-SPOT.TB ( $1.7 \%$ vs $2.0 \%[P=.895]$ ) (Fig 2$)$. In subjects with available paired test results, children $<4$ years of age had indeterminate IGRA results significantly more frequently with QFT-G and QFT-IT than with T-SPOT.TB (38.1\% vs $0 \%[P=.008]$ and $27.7 \%$ vs $2.8 \%$ [ $P=.001]$, respectively) (Fig 3, upper panel); this difference was not observed in subjects $\geq 4$ years of age $(4.5 \%$ vs $3.0 \%[P=$ $.650]$ and $3.2 \%$ vs $0 \%[P=.001]$, respectively) (Fig 3, lower panel).

The correlation between the frequency of indeterminate results and age, considered as a continuous variable, was statistically significant for both QFT-G (OR: 0.79 [95\% CI: 0.69-0.90]) and QFT-IT (OR: 0.68 [95\% CI: $0.52-0.90]$ ), but not for T-SPOT.TB (OR: 0.89 [95\% CI: 0.67-1.18]). These results did not change after adjusting for TST results, BCG status, or gender (data not shown). We also evaluated the correlation between age and the concentration of phytohaemagglutinin-induced IFN- $\gamma$ response in IGRA positive-control wells, consid- 


\begin{tabular}{|c|c|c|c|c|c|}
\hline \multirow[t]{2}{*}{ TABLE 4} & \multicolumn{5}{|c|}{$\begin{array}{l}\text { Concentrations of IFN- } \gamma \text { in the Positive-Control Wells of } \\
\text { IGRAs }\end{array}$} \\
\hline & & All & $<4 y$ & $\geq 4 y$ & $P$ \\
\hline \multicolumn{2}{|c|}{ QFT-G, $n, I U / m L$} & 181 & 39 & 142 & \\
\hline \multicolumn{2}{|c|}{ Median } & 3.01 & 1.01 & 3.73 & $<.0001$ \\
\hline \multicolumn{2}{|l|}{ Mean } & 0.86 & -0.44 & 1.22 & $<.0001$ \\
\hline \multicolumn{2}{|c|}{ SD } & 1.62 & 1.84 & 1.36 & \\
\hline \multicolumn{2}{|c|}{ Mean difference } & & & -1.65 & \\
\hline \multicolumn{2}{|c|}{ QFT-IT, n, IU/mL } & 315 & 65 & 250 & \\
\hline \multicolumn{2}{|c|}{ Median } & 3.10 & 0.75 & 3.34 & $<.0001$ \\
\hline \multicolumn{2}{|l|}{ Mean } & 2.61 & 0.68 & 3.11 & $<.0001$ \\
\hline \multicolumn{2}{|l|}{ SD } & 1.44 & 1.56 & 0.87 & \\
\hline \multicolumn{2}{|c|}{ Mean difference } & & & -2.43 & \\
\hline \multicolumn{2}{|c|}{ T-SPOT.TB, $n$, SFUs per well } & 154 & 57 & 97 & \\
\hline \multicolumn{2}{|c|}{ Median } & 103 & 142 & 87 & $<.0001$ \\
\hline \multicolumn{2}{|l|}{ Mean } & 4.65 & 4.93 & 4.48 & .004 \\
\hline \multicolumn{2}{|l|}{ SD } & 0.94 & 0.67 & 1.03 & \\
\hline \multicolumn{2}{|c|}{ Mean difference } & & & 0.45 & \\
\hline
\end{tabular}

The minimum amount of IFN- $\gamma$ in the positive-control well of QFT assays for a valid result was $0.50 \mathrm{IU} / \mathrm{mL}$ (after subtraction of the nil value). Mean and SD values were calculated on the log scale.

ered as both binary and continuous variables. Linear regression showed that the log-transformed IFN- $\gamma$ response to phytohaemagglutinin significantly increased with age in both QFT-G $(\beta=.13$ [95\% CI: $0.10-0.17]$ $P<.001)$ and QFT-IT $(\beta=.16$ [95\% CI: $0.14-0.18] P<$ $.001)$; on the contrary, the response to log-transformed IFN- $\gamma$ response to phytohaemagglutinin in T-SPOT.TB significantly decreased with age $(\beta=-.04$ [95\% CI: -.06 to -.01$] ; P=.005)$.

Considering age as a binary variable $(<4$ and $\geq 4$ years of age), a significantly lower concentration of phytohaemagglutinin-produced IFN- $\gamma$ was observed among younger children for both QFT assays, whereas this was not the case for T-SPOT.TB (Table 4). In addition, significantly higher concentrations of IFN- $\gamma$ were obtained in the positive-control wells of QFT-IT compared with QFT-G in all age classes ( $<4$ years of age: 5.4 vs 2.1 $\mathrm{IU} / \mathrm{mL}[P=.013] ; \geq 4$ years of age: $25.6 \mathrm{vs} 6.1 \mathrm{IU} / \mathrm{mL}$ $[P<.001])$ (Table 4$)$. Four of $37(10.8 \%)$ indeterminate QFT assays were in children with a positive TST: of these, 3 were BCG-vaccinated and 1 was diagnosed with $M y$ cobacterium avium infection. Among children with an indeterminate QFT test and available T-SPOT.TB test $(n=23)$, all but 1 were T-SPOT.TB-negative, and none was indeterminate with T-SPOT.TB.

Stratification of the results based on the extent of exposure to $M$ tuberculosis (ie, household or school contacts of smear-positive cases versus casual contacts or recent immigrants) showed no differences in the rates of indeterminate results for any assay (QFT-G: $P=.924$; QFT-IT: $P=.654$; T-SPOT.TB: $P=.974$ ).

\section{DISCUSSION}

Our investigation of the routine use of IGRAs in children indicates that the ELISA-based assays (QFT-G and QFT-IT) are significantly more affected by indeterminate results, because of low mitogen response, than is the ELISPOT-based test (T-SPOT.TB). This difference is particularly evident in children $<4$ years of age and is confirmed by the finding of a statistically significant inverse relationship between the amount of IFN- $\gamma$ and age for both QFT assays. This phenomenon was not observed in the T-SPOT.TB assay, in which significantly higher SFUs values were found in younger children. Other groups have explored the issue of IGRA performance in children with partially contrasting results. Connell et $\mathrm{al}^{13}$ reported a $17 \%$ incidence of indeterminate QFT-G results, which is similar to our findings. The same group observed a higher rate of indeterminate results with T-SPOT.TB than with QFT-IT. However, all of the T-SPOT.TB assays were invalid owing to high background in the negative-control well or low-cell counts. ${ }^{18}$ Mandalakas et $\mathrm{al}^{17}$ reported no indeterminate results with either QFT-G or T-SPOT.TB in a small cohort of HIVinfected children, whereas Tsiouris et $\mathrm{al}^{21}$ did not detect any indeterminate QFT-IT results in children $>5$ years of age. Our findings, based on a larger sample size, confirm that indeterminate QFT-IT results in older children are uncommon. Finally, a recent study of 227 children between 0 and 15 years of age also reported a statistically significant correlation between mitogen response in the QFT-IT assay and age. ${ }^{22}$ The variability of such findings across different studies might be explained by a mix of different factors, including the criteria used to define an indeterminate IGRA result (low mitogen versus high nil), the study design ("routine" vs "controlled" conditions), and the population tested (effect of different age groups). Certainly, this is an area where focused, prospective controlled studies are needed.

QFT-IT and T-SPOT.TB have both been approved for clinical use by the US Food and Drug Administration, although with some minor modifications in the interpretation of T-SPOT.TB results, compared with the European package insert. This reflects the vast amount of published data available. Therefore, it is predictable that IGRAs will rapidly become routine in clinical practice. At that point, differences with the standard TST will be particularly relevant. One such difference is the 3-way response of IGRAs (positive, negative, or indeterminate/ invalid), compared with the 2-way response of the TST test (positive or negative). However, the meaning and the impact of indeterminate IGRA results in clinical practice have not been properly evaluated. For now, these results should caution clinicians to be aware of potential false-negative results. In addition, although the difference in the frequency of indeterminate results between the QFTs and T-SPOT.TB in our study was striking, the clinical impact of this difference might be relatively small, because almost all indeterminate QFT results were negative with T-SPOT.TB. Another interesting finding of our study is the significantly higher concentration of IFN- $\gamma$ produced in QFT-IT compared with QFT-G. Whether this is a consequence of the in-tube format or some other technical factor (such as shaking of tubes), and whether it has an impact on antigen-specific responses, merits additional attention. 


\section{CONCLUSIONS}

Our findings, based on the retrospective evaluation of a large cohort of children, lend support to the notion that different IGRAs for the diagnosis of LTBI may produce significantly different rates of indeterminate results in young children. In particular, although both QFT formats were negatively affected by young age in terms of mitogen-stimulated IFN- $\gamma$ production, T-SPOT.TB-positive controls seemed to be equally efficient at any age. Because young children are a high-risk group and a main target for preventive LTBI treatment, these results may have an impact on the strategies aimed at TB control.

\section{REFERENCES}

1. Khan EA, Starke JR. Diagnosis of tuberculosis in children: increased need for better methods. Emerg Infect Dis. 1995; 1(4): $115-123$

2. Lewinsohn DA, Gennaro ML, Scholvinck L, Lewinsohn DM. Tuberculosis immunology in children: diagnostic and therapeutic challenges and opportunities. Int J Tuberc Lung Dis. 2004; $8(5): 658-674$

3. Newton SM, Brent AJ, Anderson S, Whittaker E, Kampmann B. Paediatric tuberculosis. Lancet Infect Dis. 2008;8(8):498-510

4. Miyawaki T, Seki H, Taga K, Sato H, Taniguchi N. Dissociated production of interleukin- 2 and immune $\gamma$ interferon by phytohaemagglutinin stimulated lymphocytes in healthy infants. Clin Exp Immunol. 1985;59(2):505-51 1

5. Huebner RE, Schein MF, Bass JB Jr. The tuberculin skin test. Clin Infect Dis. 1993;17(6):968-975

6. Steiner P, Rao M, Victoria MS, Jabbar H, Steiner M. Persistently negative tuberculin reactions: their presence among children with culture positive for Mycobacterium tuberculosis (tuberculinnegative tuberculosis). Am J Dis Child. 1980;134(8):747-750

7. Richeldi L. An update on the diagnosis of tuberculosis infection. Am J Respir Crit Care Med. 2006; 174(7):736-742

8. Pai M, Zwerling A, Menzies D. Systematic review: T-cell-based assays for the diagnosis of latent tuberculosis infection: an update. Ann Intern Med. 2008;149(3):177-184

9. Diel R, Loddenkemper R, Meywald-Walter K, Niemann S, Nienhaus A. Predictive value of a whole blood IFN-gamma assay for the development of active tuberculosis disease after recent infection with Mycobacterium tuberculosis. Am J Respir Crit Care Med. 2008; 177(10):1164-1170

10. Bakir M, Millington KA, Soysal A, et al. Prognostic value of a T-cell-based, interferon- $\gamma$ biomarker in children with tuberculosis contact. Ann Intern Med. 2008;149(11):777-787

11. Group PTC. Targeted tuberculin skin testing and treatment of latent tuberculosis infection in children and adolescents. Pediatrics. 2004;114(4):1175-1201

12. Dogra S, Narang P, Mendiratta DK, et al. Comparison of a whole blood interferon-gamma assay with tuberculin skin testing for the detection of tuberculosis infection in hospitalized children in rural India. $J$ Infect. 2007;54(3):267-276

13. Connell TG, Curtis N, Ranganathan SC, Buttery JP. Performance of a whole blood interferon gamma assay for detecting latent infection with Mycobacterium tuberculosis in children. Thorax. 2006;61(7):616-620

14. Ferrara G, Losi M, D'Amico R, et al. Use in routine clinical practice of two commercial blood tests for diagnosis of infection with Mycobacterium tuberculosis: a prospective study. Lancet. 2006;367(9519):1328-1334

15. Ferrara G, Losi M, Meacci M, et al. Routine hospital use of a commercial whole blood interferon- $\gamma$ assay for tuberculosis infection. Am J Respir Crit Care Med. 2005;172(5):631-635

16. Richeldi L, Ewer K, Losi M, et al. T cell-based tracking of multidrug resistant tuberculosis infection after brief exposure. Am J Respir Crit Care Med. 2004;170(3):288-295

17. Mandalakas AM, Hesseling AC, Chegou NN, et al. High level of discordant IGRA results in HIV-infected adults and children. Int J Tuberc Lung Dis. 2008;12(4):417-423

18. Connell TG, Ritz N, Paxton GA, Buttery JP, Curtis N, Ranganathan SC. A three-way comparison of tuberculin skin testing, QuantiFERON-TB gold and T-SPOT.TB in children. PLOS ONE. 2008;3(7):e2624

19. Kampmann B, Tena-Coki G, Anderson S. Blood tests for diagnosis of tuberculosis. Lancet. 2006;368(9532):282

20. American Thoracic Society. Diagnostic standards and classification of tuberculosis in adults and children. Am J Respir Crit Care Med. 2000;161(4 pt 1):1376-1395

21. Tsiouris SJ, Austin J, Toro P, et al. Results of a tuberculosisspecific IFN-gamma assay in children at high risk for tuberculosis infection. Int J Tuberc Lung Dis. 2006;10(8):939-941

22. Chun JK, Kim CK, Kim HS, et al. The role of a whole blood interferon gamma assay for the detection of latent tuberculosis infection in Bacille Calmette-Guerin vaccinated children. Diagn Microbiol Infect Dis. 2008;62(4):389-394 


\section{Performance of Commercial Blood Tests for the Diagnosis of Latent Tuberculosis Infection in Children and Adolescents}

Barbara Maria Bergamini, Monica Losi, Francesca Vaienti, Roberto D'Amico, Barbara Meccugni, Marisa Meacci, Donatella De Giovanni, Fabio Rumpianesi,

Leonardo M. Fabbri, Fiorella Balli and Luca Richeldi

Pediatrics 2009;123; 419

DOI: $10.1542 /$ peds. $2008-1722$

\section{Updated Information \& Services}

References

Citations

Subspecialty Collections

Permissions \& Licensing

Reprints including high resolution figures, can be found at: /content/123/3/e419.full.html

This article cites 22 articles, 2 of which can be accessed free at:

/content/123/3/e419.full.html\#ref-list-1

This article has been cited by 15 HighWire-hosted articles: /content/123/3/e419.full.html\#related-urls

This article, along with others on similar topics, appears in the following collection(s):

Infectious Disease

/cgi/collection/infectious_diseases_sub

Information about reproducing this article in parts (figures, tables) or in its entirety can be found online at:

/site/misc/Permissions.xhtml

Information about ordering reprints can be found online: /site/misc/reprints.xhtml

PEDIATRICS is the official journal of the American Academy of Pediatrics. A monthly publication, it has been published continuously since 1948. PEDIATRICS is owned, published, and trademarked by the American Academy of Pediatrics, 141 Northwest Point Boulevard, Elk Grove Village, Illinois, 60007. Copyright @ 2009 by the American Academy of Pediatrics. All rights reserved. Print ISSN: 0031-4005. Online ISSN: 1098-4275.

\section{American Academy of Pediatrics}




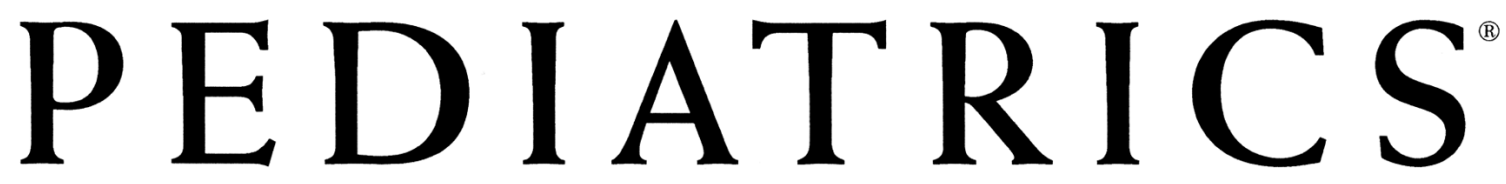

OFFICIAL JOURNAL OF THE AMERICAN ACADEMY OF PEDIATRICS

\section{Performance of Commercial Blood Tests for the Diagnosis of Latent Tuberculosis Infection in Children and Adolescents}

Barbara Maria Bergamini, Monica Losi, Francesca Vaienti, Roberto D'Amico, Barbara Meccugni, Marisa Meacci, Donatella De Giovanni, Fabio Rumpianesi, Leonardo M. Fabbri, Fiorella Balli and Luca Richeldi Pediatrics 2009;123; 419

DOI: $10.1542 /$ peds.2008-1722

The online version of this article, along with updated information and services, is located on the World Wide Web at: /content/123/3/e419.full.html

PEDIATRICS is the official journal of the American Academy of Pediatrics. A monthly publication, it has been published continuously since 1948. PEDIATRICS is owned, published, and trademarked by the American Academy of Pediatrics, 141 Northwest Point Boulevard, Elk Grove Village, Illinois, 60007. Copyright (c) 2009 by the American Academy of Pediatrics. All rights reserved. Print ISSN: 0031-4005. Online ISSN: 1098-4275.

\section{American Academy of Pediatrics}

